\title{
Praha, Národní Knihovna České Republiki, XXV.C.31
}

\author{
Costantinopoli?, XIII-XIV sec.
}

ff. 168 [+166, 55 bis, 127 bis]; num. recente nel marg. inf. est. a matita, erronea nel numero 142 segnato come 146; cart. senza filigrana; $1^{4}(4), 2^{10-2}(12), 3^{8}(20) 4^{6+1}(27), 5-7^{8}(51), 7^{6+2}$ (58), 8-16 $6^{8}(129), 17^{6+1}(136), 18^{8-2}(142), 19-21^{8}$ (166); fasc. assente; ff 1r-11v: mm $239 \times 150=$ 20 // 185 // $34 \times 10$ // 110 // 30; 1l. 59, unità di rigatura mm 3, rigatura tracciata a secco sul recto di alcuni fogli, tracciate solo le linee verticali atte a delimitare lo spazio scrittorio (f. 7r); ff. 12r-136v: mm $236 \times 153=19 / / 178 / / 43 \times 16 / / 110 / / 28 ; 11.60$, unità di rigatura mm 3, rigatura assente (f. 19r); ff. 137r-168v: mm $239 \times 153=20$ // 178 // $40 \times 12$ // 112 // 28; 1l. 31, unità di rigatura mm 5, rigatura assente (f. 157r). È bianco il f. 122v. Legatura bizantina formata da assi di legno, ricoperte da cuoio impresso a freddo; sul piatto anteriore e posteriore è presente un motivo a cornici rettangolari incastrate l'una nell'altra, ognuna delle quali è realizzata a ferri impressi: la prima e la terza cornice presentano un motivo geometrico-floreale, la seconda floreale. Nel rettangolo centrale si alternano verticalmente quattro ferri a losanga e quattro ferri circolari, contenenti rispettivamente un giglio e un'aquila. Sul piatto anteriore resta traccia dell'originaria presenza di due fermagli. Sulla rete del dorso, visibile a causa della perdita del cuoio, si legge l'etichetta con i numeri 60 o 61. Sui contropiatti, anteriore e posteriore, prove di scrittura.

\section{Scrittura}

Nel codice si distinguono quattro mani: <Isacco Argiro (Mondrain 2007, 169; Pérez Martín 2009, 338)> (ff. 1r-11v); mano B (ff. 12r-121v, 1. 12, 123r-136v), riferibile alla fine del XIII secolo; <Gregorio dei Mangani (Pérez Martín 2009, 338)> (ff. 118v, 1. 39-119r, 1. 14, 137r, 11. 1-8), intervenuto per completare due vacua; mano D (ff. 137r, 1. 9-140r, 1. 25, 141r-166v), riferibile alla prima metà del XIV.

\section{Decorazione}

Titoli e decorazioni in inchiostro rosso.

\section{Interventi successivi}

<Giorgio Galesiota (Pérez Martín 2009, 339)> (f. 122r, f. 140r, 1l. 27-32; f. 138r, nel margine superiore; f. 140v) copia, sui fogli rimasti bianchi e probabilmente dopo le altre integrazioni di XIV sec., alcuni epigrammi e estratti. 


\section{Contenuto}

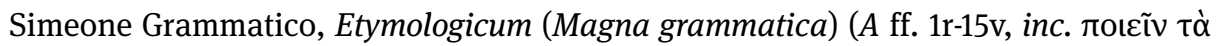

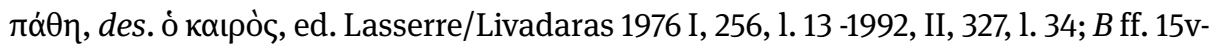

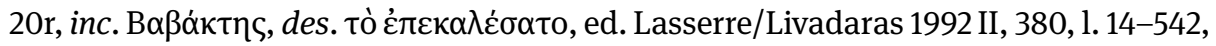

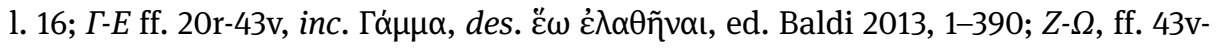

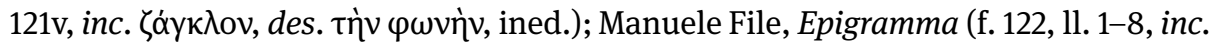

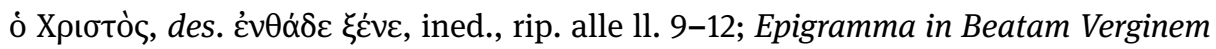

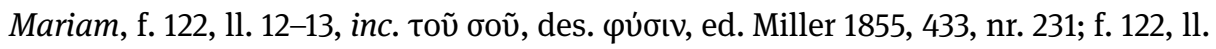

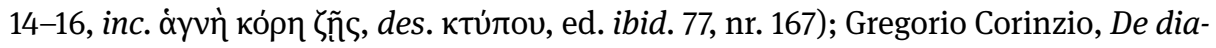

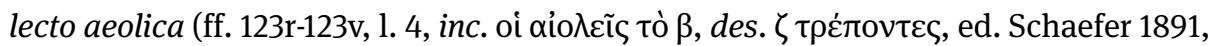
572-613, 1. 4); Giorgio Cherobosco, De tropis poeticis in recensione breviore (ff. 123v, 1.

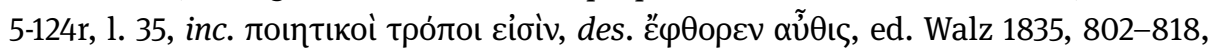
1. 16); Anonimo, Scholiis in Dionysii Thracis Artem grammaticam selectum (f. 124v, 11.

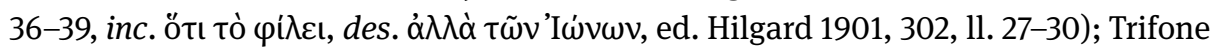

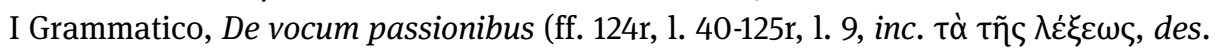

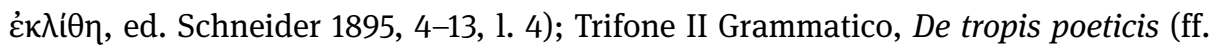

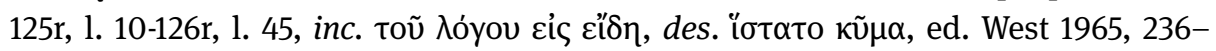

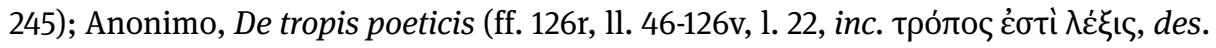

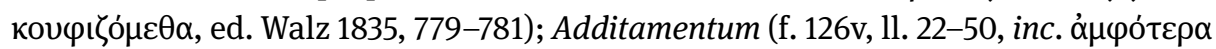

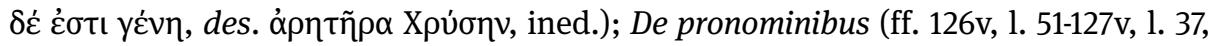

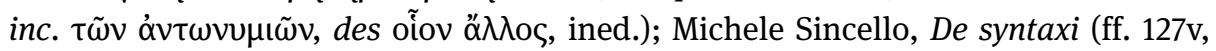

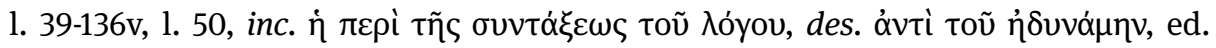
Donnet 1982, 159-413, 1. 1739); Polibio Sardiano, Excerpta e De soloecismo (ff. 137r-

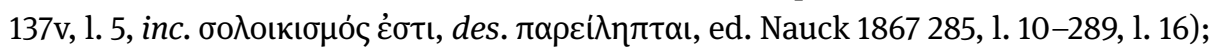

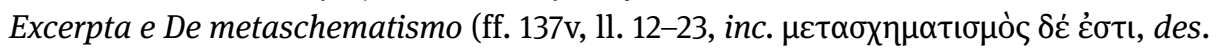

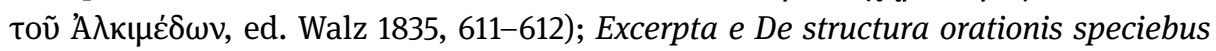

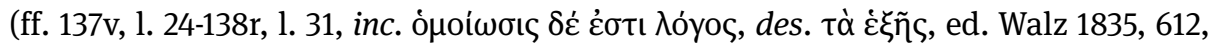

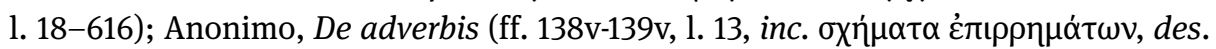

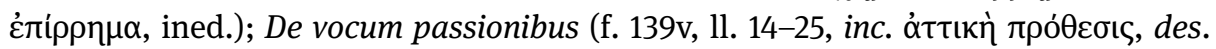

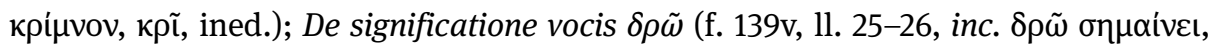

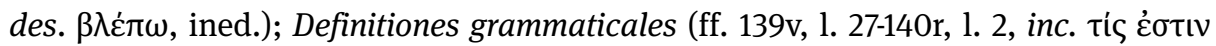

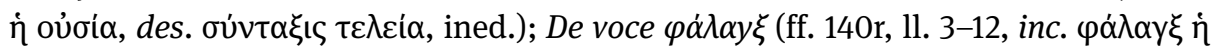

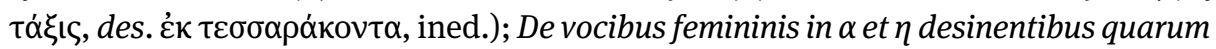

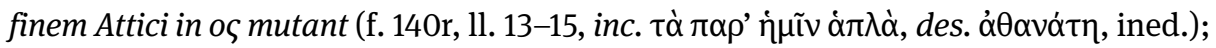

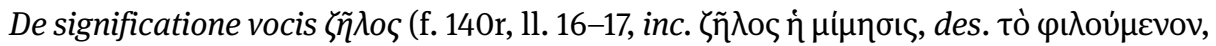

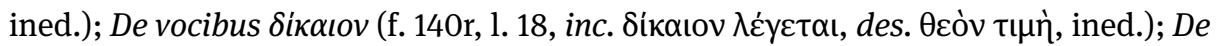

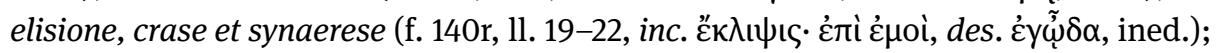

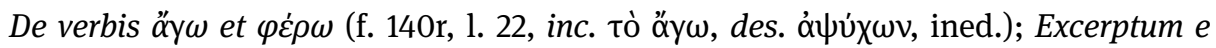

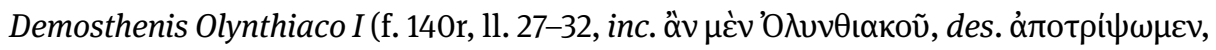
ed. Fuhr 1914, 12, 1. 23-13, 1. 8); Manuele File, Epigrammata (f. 140v, 1l. 2-3, inc. åxદı

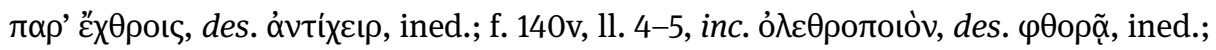


Epigramma in Lucam, f. 140v, 1l. 7-8, inc. $\tilde{\omega} \mu o ́ \sigma \chi \varepsilon, ~ \Lambda o v \kappa \tilde{a, ~ d e s . ~ Ө \eta \sigma \alpha v \rho i ́ \sigma \omega, ~ e d . ~ M i l l e r ~}$

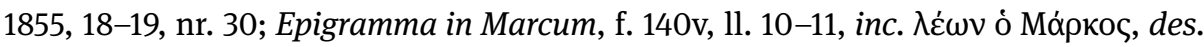
ßpóxous, ed. ibid. 21, nr. 38; Epigramma in Joannem, f. 140v, 1l. 13-14, inc. Tòv ảctóv

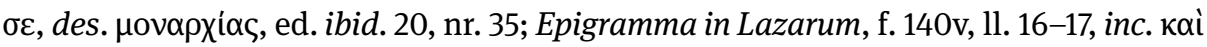

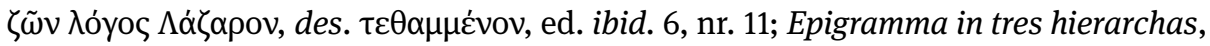

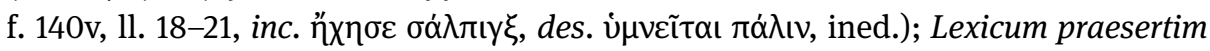

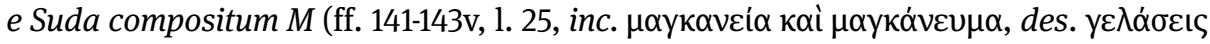

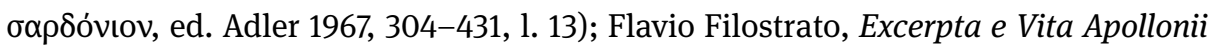

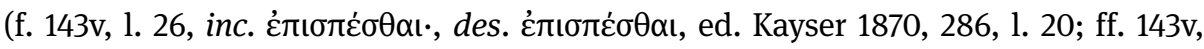

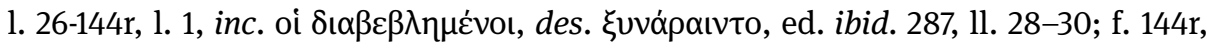

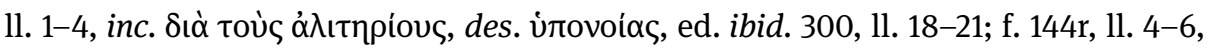

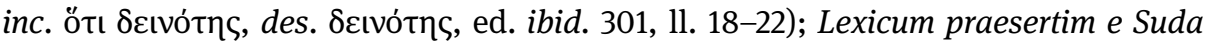

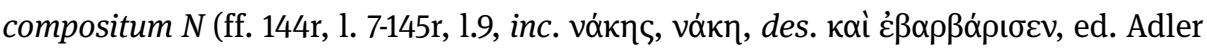
1967, 435, 1. 6-489, 1. 14); Flavio Filostrato, Excerpta e Vita Apollonii (f. 145r, 1l. 10-13,

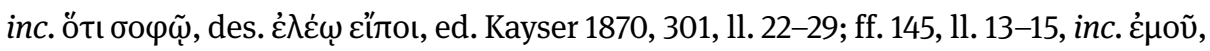
des. $\mu \alpha \dot{\lambda}\lrcorner \sigma \tau \alpha$, ed. ibid. 304, 11. 14-17); Lexicum praesertim e Suda compositum $\Xi$ (ff. 145r,

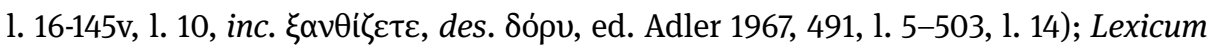

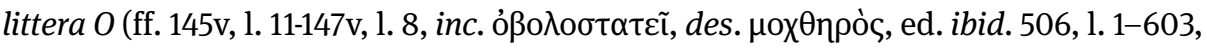

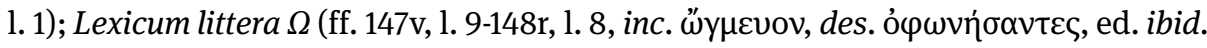
604, 1. 21-632, 1. 9); Flavio Filostrato, Excerpta e Vita Apollonii (f. 148r, 11. 9-12, inc. ö ı

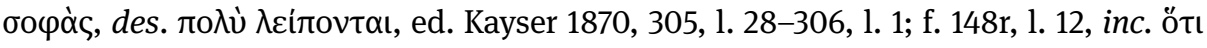

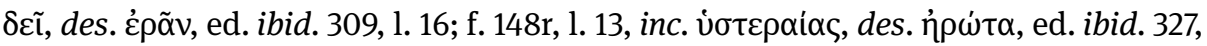

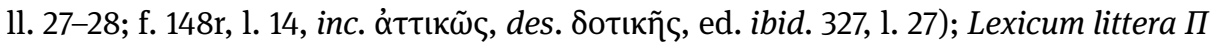

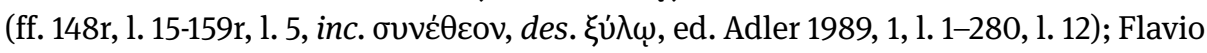

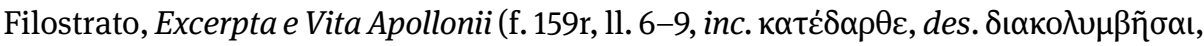

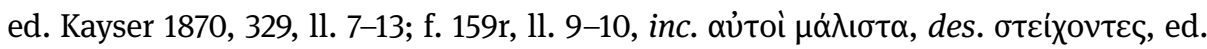

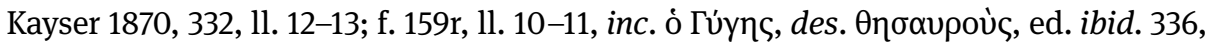

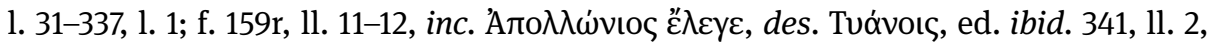

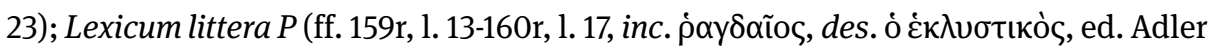

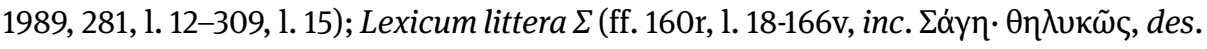
өopúßou kai, ed. ibid. 311, 1. 26-478, 1. 17).

\section{Relazioni stemmatiche}

Insieme ai codici S. Marco 303 e Leid. Voss. gr. Q.20, il manoscritto Prah. XXV.C. 31 è uno dei tre testimoni che tramandano l'Etymologicum Symeonis nella redazione nota come Magna Grammatica, per la quale il codice di Praga (P) discende, insieme con il manoscritto S. Marco 303 (C), dal subarchetipo $\delta$ (Baldi 2013, XXV-XXVI). Per quanto riguarda gli excerpta della Vita di Apollonio di Filostrato, il testimone è stato messo in relazione con i codici Marc. gr. XI.29, Par. gr. 1696 e Scorial. Ф.III.8; presenta infatti in un estratto una lezione comune a questi manoscritti (Boter 2014, 34). 


\section{Storia del codice}

Il codice, presente nella biblioteca del Prodromo di Petra nel 1841, quando Minoïde Mynas ne ha fornito una descrizione nel suo catalogo (Papazoglou 1991, 74-79, nr. 96), è stato trafugato durante il raid del 28/29 giugno del 1917 ad opera delle truppe bulgare comandate da Vladimír Sís ed è giunto così nella biblioteca di Národní Knihovna (Olivier/Monégier du Sorbier 2006, 221).

\section{Bibliografia}

\section{Cataloghi}

Olivier/Monégier du Sorbier (2006) 205-221; Papazoglou (1991) 74-79, nr. 96.

\section{Edizioni}

Adler (1967); Adler (1989); Baldi (2013); Donnet (1982); Fuhr (1914); Miller (1855); Nauck (1867); Kayser (1870); Hilgard (1901); Lasserre/Livadaras (1976) I; Lasserre/Livadaras (1992) II; Schaefer (1891); Schneider (1895); West (1965); Walz (1835).

\section{Codicologia e paleografia}

Baldi (2014) 367; Boter (2014) 33-34; Galán Vioque (2013) 96; Mondrain (2007) [2008] 163; Pérez Martín (2009) 338-339.

\section{Riproduzioni}

Olivier/Monégier du Sorbier (2006) tavv. XIX-XXIV. 